\title{
Affinity Study of Novel Gelatin Cell Carriers
}

\section{for Fibronectin}

S. Van Vlierberghe ${ }^{1}$, E. Vanderleyden ${ }^{1}$, P. Dubruel ${ }^{1}$, F. De $\operatorname{Vos}^{2}$, E. Schacht ${ }^{1, *}$

${ }^{1}$ Polymer Chemistry \& Biomaterials Research Group, Ghent University, Ghent, Belgium

${ }^{2}$ Department of Pharmaceutical Analysis, Ghent University, Ghent, Belgium

*Corresponding author: Etienne Schacht

Krijgslaan 281 (Building S4 Bis)

B-9000 Ghent

e-mail: Etienne.Schacht@UGent.be

tel: 003292644497

fax: 003292644972

Keywords: hydrogels, proteins, quartz crystal microbalance, radiolabelling, surface plasmon resonance 


\section{Summary}

In the present work, the affinity of gelatin type A and B for fibronectin (Fn) was evaluated using surface plasmon resonance, quartz crystal microbalance and radiolabelling experiments. The affinity study was performed on both hydrogel films and 3D scaffolds. The results indicate that type A gelatin films possess a higher affinity for Fn compared to type B gelatin films. This can be explained by a combination of hydrophobic and electrostatic interactions occurring between gelatin type A and Fn. In a second part of the work, the affinity of Fn for a series of porous gelatin scaffolds was also evaluated. The scaffolds were prepared by a cryogenic treatment and subsequent freeze-drying (Van Vlierberghe et al, 2007, Biomacromolecules, 8, 331-337). Type I hydrogels were composed of cone-like pores with decreasing diameter from top $(330 \mu \mathrm{m})$ to bottom $(20-30 \mu \mathrm{m})$. Type II scaffolds contained spherical pores with an average diameter of $135 \mu \mathrm{m}$. The results of the various scaffolds indicate that the Fn density on the gelatin scaffolds can be easily finetuned by varying the Fn concentration, the gelatin type (A versus B) and various scaffold properties including the pore size and the pore geometry (type I versus type II scaffolds). 


\section{Introduction}

Fibronectin $(\mathrm{Fn})$ is a major component of the extracellular matrix. ${ }^{[1]}$ In a surface adsorbed state, it is active and regulates a wide variety of cellular processes including cell adhesion, differentiation, proliferation and migration. ${ }^{[2]}$ Consequently, Fn is often applied as a bioactive coating in order to improve the cell interactive properties of biomaterials. ${ }^{[3-5]}$ In many cell interaction studies, Fn-coated surfaces were applied to enhance cell attachment. ${ }^{[6,7]}$ Moreover, the effect is Fn concentration dependent. ${ }^{[8]}$ In one study, Ti surfaces, coated with a Fn concentration of $0.21 \mu \mathrm{g} / \mathrm{mm}^{2}$, significantly increased cell attachment in contrast to uncoated surfaces and Ti surfaces, containing $0.023 \mu \mathrm{g} / \mathrm{mm}^{2}$ or $0.65 \mu \mathrm{g} / \mathrm{mm}^{2} \mathrm{Fn} \cdot{ }^{[9]}$ It was suggested that Fn affects cell attachment by binding through cell surface receptors and mediating adhesive interactions. It was also reported that Fn unfolds into an inactive conformation at a low concentration. However, at high concentrations, the unfolding was prevented by molecule packing phenomena. These data suggest that the conformation of proteins plays an important role in mediating cell attachment. ${ }^{[9,10]}$ In another study, it was shown that the Fn densities required to enable cell attachment on culture plastics was in the range of $10-15 \mathrm{ng} / \mathrm{cm}^{2}{ }^{[11]}$ As a consequence, controlling and varying the amount of Fn deposited onto biomaterials is essential to enable the preparation of tissue engineering devices possessing specific cellinteractive properties (e.g. for cardiovascular, ocular or skin replacement applications).

In a previous study, we have reported the synthesis of porous gelatin scaffolds with a controlled pore size and pore morphology by applying a cryogenic treatment. ${ }^{[12]}$ The porous hydrogels were suitable for the attachment and growth of a large variety of human cells over longer time periods. ${ }^{[13]}$ In the present work, Fn will be applied as coating on these scaffolds to further improve their applicability for tissue engineering purposes. 
The concept is based on the fact that several subunits of Fn possess a binding site for gelatin. ${ }^{[14]}$ This pronounced affinity has resulted previously in the application of gelatinmodified stationary phases for the chromatographic separation of Fn from plasma and serum. ${ }^{[15-17]}$ In addition, Miekka et al have shown that the gelatin-Fn bond is rather strong. ${ }^{[18]}$ Based on these literature data, gelatin-based hydrogels are anticipated to bind Fn.

Since chemical modifications of Fn can alter its biological activity, incorporation via physical interactions is preferred. ${ }^{[19,20]}$ The coating process will be evaluated using surface plasmon resonance (SPR), quartz crystal microbalance (QCM) and radiolabelling experiments. Each of these techniques has specific advantages and drawbacks which are summarised in table 1.

A quantitative evaluation of the Fn affinity for gelatin hydrogels has, to the best of our knowledge, not been published so far. Therefore, in the present work, we aim to apply and compare various methods for studying the effect of the gelatin type (A versus B), the material structure (film versus porous scaffold) as well as the effect of pore size and geometry on the interaction with Fn. 


\section{Experimental Part}

\subsection{Materials}

Type $\mathrm{B}$ gelatin $(\mathrm{IEP}=5$, Bloom strength $=257)$, isolated from bovine skin by the alkaline process, was kindly supplied by SKW Biosystems (Ghent, Belgium). Type A gelatin (IEP = 8.8, Bloom strength $=202$ ), isolated from porcine skin by the acid treatment, was obtained from Rousselot (Ghent, Belgium). Methacrylic anhydride (MAA) was purchased from Aldrich (Bornem, Belgium) and was used as received. Dialysis membranes Spectra/Por® 4 (MWCO 12,000-14,000 Da) were obtained from Polylab (Antwerp, Belgium). 1-[4-(2Hydroxyethoxy)-phenyl]-2-hydroxy-2-methyl-1-propane-1-one (Irgacure® 2959) was a kind gift from Ciba Speciality Chemicals N.V. (Groot-Bijgaarden, Belgium). An LWUV-lamp model VL-400L (Vilber Lourmat, Marne La Vallée, France) was used for sample curing. Fibronectin $(\mathrm{Fn})$ from bovine plasma $(0.1 \%$ solution, $1 \mathrm{mg} / \mathrm{ml}$ in $0.5 \mathrm{M} \mathrm{NaCl}, 0.05 \mathrm{M}$ Tris and $\mathrm{pH}$ 7.5) was purchased from Sigma-Aldrich (Bornem, Belgium). Polyclonal rabbit antihuman Fn solution (5.1 g/l) was obtained from DakoCytomation (Heverlee, Belgium). As demonstrated by rocket immunoelectrophoresis, the antibody cross-reacts with the fibronectin-equivalent protein in cat, cow, deer, dog, elephant, goat, guinea pig, hamster, hen, horse, mink, monkey, mouse, musk ox, polecat, racoon dog, rat, seal, sheep, swine and wildebeest. $^{[21]}$

$\mathrm{Na}^{125} \mathrm{I}(100 \mathrm{mCi} / \mathrm{ml})$ was purchased from Perkin-Elmer. Sodium phosphate (dibasic, anhydrous, p.a.) and potassium hydrogen phosphate were obtained from Acros (Geel, Belgium). Sodium azide (99\%) was obtained from Avocado Research Chemicals Ltd. (Karlsruhe, Germany).

The SPR chips used were bare gold chips (SIA Au Kit, Biacore, GE Healthcare Europe GmbH, Belgium). 


\subsection{Preparation of hydrogel films}

Both type A and type B gelatin-based hydrogel films were prepared as described previously. ${ }^{[22]}$ Briefly, the amine sidegroups in gelatin were chemically modified with methacrylamide moieties, resulting in a degree of substitution (DS) of $60 \%$ relative to the original content of the primary amines. ${ }^{[22]}$ In a typical experiment, an aqueous solution of modified gelatin $(10 \mathrm{w} / \mathrm{v} \%$ gel-MOD), containing the photo-initiator Irgacure® 2959 (2 mol\% relative to the methacrylamide side chains), was injected between two glass plates separated by a silicone spacer ( $1 \mathrm{~mm}$ thick). Subsequently, the mixture was exposed to UVlight $\left(279 \mathrm{~nm}, 10 \mathrm{~mW} / \mathrm{cm}^{2}\right)$ for 30 minutes. The chemically crosslinked hydrogels were stored at $5^{\circ} \mathrm{C}$ until evaluation.

\subsection{Preparation of porous hydrogel scaffolds}

Both type A and type B gelatin-based scaffolds were prepared as described previously. ${ }^{[12]}$ Shortly, methacrylamide modified gelatin (10 w/v \%), containing 2 mol\% Irgacure® 2959, was allowed to gel prior to exposure to UV-light $\left(279 \mathrm{~nm}, 10 \mathrm{~mW} / \mathrm{cm}^{2}\right)$. After placing the chemically cross-linked hydrogel in the cryo-unit (see figure 1), the temperature of freezing and the cooling rate were programmed with a Julabo, type FP40-ME. Under the bottom of the mould, a Peltier element (thermo electric cooler, TEC) was positioned to enable a temperature gradient to be applied. The applied TEC was a DuraTec DT12 type from Marlow industries. The aluminum heat exchangers and the electronic TEC controller were designed, built and assembled by the technical workshop (CWFW) of the Faculty of Sciences, Ghent University. Two scaffold types were prepared by applying different cooling regimes (see table 2). Type I hydrogels, possessing channel-like pores, were cooled from $21^{\circ} \mathrm{C}$ to $-30^{\circ} \mathrm{C}$ at a cooling rate of $0.15^{\circ} \mathrm{C} / \mathrm{min}$. During the freezing step, a temperature gradient of $30^{\circ} \mathrm{C}$ between top and bottom 
of the scaffold was applied. Type II hydrogels were obtained by applying the same cooling regime as for type I hydrogels, but without implementing a temperature gradient during the cryogenic treatment. After incubating the samples for one hour at the final freezing temperature (i.e. $-30^{\circ} \mathrm{C}$ ), the frozen hydrogels were transferred to a freeze-dryer to remove the ice crystals, resulting in porous scaffolds. ${ }^{[12]}$

\subsection{Water uptake capacity}

Freeze-dried gelatin scaffolds $\left(1 \times 1 \times 0.5 \mathrm{~cm}^{3}\right)$ were weighed and then immersed in $80 \mathrm{ml}$ double distilled water at $37^{\circ} \mathrm{C}$, in the presence of sodium azide to prevent bacterial growth. At regular time points, the hydrogels were removed from the solution, dipped gently with paper and weighed again. The hydration properties were calculated using the following equation:

$$
\% \text { Water uptake }=\left[\left(\mathrm{W}_{\mathrm{t}}-\mathrm{W}_{0}\right) / \mathrm{W}_{0}\right] \times 100 \%
$$

$\mathrm{W}_{0}=$ initial weight of the dry scaffold; $\mathrm{W}_{\mathrm{t}}=$ weight of the hydrated hydrogel at time point $\mathrm{t}$.

All data points are the mean value of three separate measurements.

\subsection{Measurements of the hydrodynamic volume of $F n$}

The hydrodynamic diameter of Fn was obtained using light scattering measurements (DLS700, Otsuka). The measurements were carried out at $25^{\circ} \mathrm{C}$ at a scattering angle of $90^{\circ}$ and the reported value for the hydrodynamic diameter is an average of three measurements. The Fn stock solution ( $1 \mathrm{mg} / \mathrm{ml}$ in $0.5 \mathrm{M} \mathrm{NaCl}, 0.05 \mathrm{M}$ Tris, $\mathrm{pH}$ 7.5) was diluted to a concentration of $50 \mu \mathrm{g} / \mathrm{ml}$ using double distilled water.

\subsection{Surface Plasmon Resonance experiments}

The Fn affinity for both type A and type B gelatin was monitored using surface plasmon resonance (SPR) measurements. The apparatus used was a Biacore-X equipped with a $500 \mu 1$ 
Hamilton syringe. All measurements were performed at $25^{\circ} \mathrm{C}$ using a phosphate buffer $(0.05$ $\mathrm{M}, \mathrm{pH}=7.4)$ at a flow rate of $50 \mu 1 / \mathrm{min}$. The sensor surface was spincoated using $90 \mu 1$ of an aqueous 5 w/v\% gel-MOD (degree of substitution 60\%) solution, containing 2 mol\% Irgacure ${ }^{\circledR} 2959$, at a speed of $6000 \mathrm{rpm}$ during 90 seconds prior to UV-exposure (20 min., $\left.279 \mathrm{~nm}, 10 \mathrm{~mW} / \mathrm{cm}^{2}\right)$.

After spincoating and crosslinking, the gelatin coated sensor was inserted in the SPR apparatus. After baseline stabilisation, $50 \mu \mathrm{l}$ of an aqueous Fn solution $(1-200 \mu \mathrm{g} / \mathrm{ml}, \mathrm{pH}$ 7.4) was injected while monitoring the response signal. In a final step, $50 \mu l$ of an anti-Fn solution (50 mg/l, pH 7.4) was rinsed over the sensor surface. All values reported are relative to a reference flow channel. Association and dissociation constants were obtained using the BIA evaluation software (version 3.1).

\subsection{Surface analysis}

The presence of gelatin on the sensor chip after spincoating and the stability of the applied layer after incubation in SPR running buffer, was confirmed by ATR-FTIR measurements (Biorad FT-IR spectrometer FTS 575C), atomic force microscopy (AFM), contact angle measurements and scanning electron microscopy (SEM).

AFM-studies were performed with a Nanoscope IIIa Multimode (Digital Instruments, Santa Barbara, California, USA) applying 'tapping mode' in air. Changes in surface morphology are quantified by root-mean-square (RMS) roughness values $\left(\mathrm{R}_{\mathrm{q}}\right)$. The RMS roughness is defined as follows:

$$
\mathrm{R}_{\mathrm{q}}=\sqrt{\frac{\sum\left(Z_{i}-Z_{\text {ave }}\right)^{2}}{N}},
$$

where $\mathrm{Z}_{\mathrm{ave}}$ is the average $\mathrm{Z}$ height value within a given area, $\mathrm{Z}_{\mathrm{i}}$ is the current $\mathrm{Z}$ value and $\mathrm{N}$ is the number of points within the given area. 
For the contact angle measurements, $1 \mu \mathrm{l}$ of double distilled water was placed on the hydrogel surface. The penetration of the droplet in the hydrogel film was imaged using a video camera using 25 frames/second. The contact angle was determined on the screen of the monitor using the imaging software provided by the supplier (SCA 20, version 2.1.5 build 16).

The thickness of gold-sputtered gelatin-coatings was measured using scanning electron micrographs of cross-sections of spincoated chips obtained on a Fei Quanta 200F (field emission gun) scanning electron microscope.

\subsection{Quartz Crystal Microbalance experiments}

Quartz Crystal Microbalance measurements were performed using a Q-Sense E4 apparatus (Q-Sense AB, Göteborg, Sweden). Gold-coated QCM crystals (5 MHz, AT-cut) were spincoated with $90 \mu 1$ of an aqueous $5 \mathrm{w} / \mathrm{v} \%$ gel-MOD (degree of substitution $60 \%$ ) solution, containing $2 \mathrm{~mol} \%$ Irgacure ${ }^{\circledR} 2959$, at a speed of $6000 \mathrm{rpm}$ during 90 seconds prior to UVexposure (20 min., $\left.279 \mathrm{~nm}, 10 \mathrm{~mW} / \mathrm{cm}^{2}\right)$.

Next, the gel-MOD-coated sensors were inserted in the QCM apparatus. After baseline stabilisation, aqueous Fn solutions (1 and $25 \mu \mathrm{g} / \mathrm{ml})$ were rinsed over the gel-MOD-coated surface. Next, the system was flushed with PBS (pH 7.4) until a stable baseline was obtained, followed by rinsing an aqueous anti-Fn solution $(0.051 \mathrm{~g} / \mathrm{l})$ over the sensor surface. The measurements were performed at $25^{\circ} \mathrm{C}$. Multiple frequency and dissipation data were modelled and converted to deposited masses on the crystal surface.

\subsection{Radiolabelling experiments}

Fn was labelled with ${ }^{125}$ I. Isotopes were obtained from Bristol-Myers Squibb. Radioiodination was performed using the Iodo-Gen method as previously described. ${ }^{[23]}$ In brief, a mixture of sodium iodide and protein was added to an Iodo-Gen-coated reaction vial and reacted for 15 
min at room temperature. Protein-bound iodine was separated from free iodide by passing the solution over a PD-10 column (GE Healthcare Europe) equilibrated with PBS. Finally, aqueous ${ }^{125} \mathrm{I}-\mathrm{Fn}$ solutions $(1-200 \mu \mathrm{g} / \mathrm{ml}, \mathrm{pH} 7.4)$ were prepared.

Hydrogel films $(\varnothing 7 \mathrm{~mm})$ and hydrogel scaffolds $(0.5 \mathrm{~cm}$ thick, $\varnothing 7 \mathrm{~mm})$ were incubated for 1 hour in respectively $500 \mu \mathrm{l}$ and $1 \mathrm{ml}$ phosphate buffered saline (pH 7.4) (PBS), containing ${ }^{125} \mathrm{I}-\mathrm{Fn}(1-200 \mu \mathrm{g} / \mathrm{ml})$. Next, the hydrogels were removed and washed three times in $500 \mu \mathrm{l}$ PBS for 5 minutes. The incorporated Fn amount was obtained by counting the hydrogel radioactivity with a gamma counter (Cobra II, Perkin Elmer). All experiments were performed in triplicate. 


\section{Results and Discussion}

The rationale for coating gelatin scaffolds with Fn, is based on the anticipated affinity between gelatin and fibronectin. ${ }^{[14]}$ The gelatin-Fn affinity has been previously applied in affinity chromatography using gelatin-based columns in which Fn was purified from plasma and serum. $^{[15-17]}$

In the present work, we have applied surface plasmon resonance (SPR), quartz crystal microbalance (QCM) and radiolabelling experiments to evaluate the fibronectin (Fn) affinity of gelatin. Since SPR and QCM analysis require films which are deposited on the chips, we first studied the Fn affinity for hydrogel films. For the final biomaterial application, porous 3D-scaffolds are required. So, we also included those materials in the affinity study using radiolabelling experiments. The effect of the gelatin type (A versus B), the hydrogel structure (film versus porous scaffold) and the scaffold characteristics (pore size and geometry) were studied.

\subsection{Surface plasmon resonance measurements}

\subsubsection{Study of the binding affinity}

In a first part of the work, the affinity of gelatin type B for Fn was monitored using SPR. SPR is a very sensitive technique that enables real time measurement of adhesion phenomena at interfaces. To enable SPR analysis, gelatin-methacrylamide (gel-MOD) type B was spincoated on a SPR sensor and subsequently crosslinked. In order to verify the presence of gel-MOD onto the sensor surface, ATR-IR spectroscopy, contact angle measurements and atomic force microscopy were performed. ATR-IR spectra were recorded before and after spincoating (see figure 2). It can be concluded that upon spincoating, the typical protein peaks 
$\left(1650 \mathrm{~cm}^{-1}\right.$ and $1540 \mathrm{~cm}^{-1}$ ) appeared in the spectrum, indicating the deposition of a gel-MOD layer on the surface of the sensor chip. Moreover, a decrease in contact angle after spincoating from $102^{\circ}$ to $40^{\circ}$ using $5 \mathrm{w} / \mathrm{v} \%$ gel-MOD was observed (figure 3). Next, surface profiles of both an uncoated gold chip (figure 4A) and a gel-MOD-coated sensor (figure 4B) were recorded using AFM. As indicated in figure 4, the immobilization of gel-MOD on the sensor chip significantly alters the surface morphology. The uncoated gold chip has a relatively smooth surface (figure 4A), while after gel-MOD immobilization, globular domains can be distinguished in the surface profile (figure 4B). Moreover, the roughness, as obtained from equation (1), increased from $0.1 \mathrm{~nm}$ to $0.4 \mathrm{~nm}$ after gel-MOD immobilization on the SPR chip. Stability studies further revealed that the coating applied was stable at room temperature against the running buffer of the SPR experiment (data not shown).

Since the layer deposited on the sensor surface may not exceed $300 \mathrm{~nm}$ to enable propagation of the evanescent wave in the surrounding medium, we also measured the thickness of the deposited layer. In figure 5, an image showing a cross-section of a gel-MOD-coated sensor as obtained using scanning electron microscopy (SEM) is depicted. The result indicates that the layer thickness was $60 \mathrm{~nm}$, thus enabling the gel-MOD-coated sensor to be applied in SPR measurements.

The SPR sensorgrams (see figure 6A) indicate that the adsorbed amount of Fn increased with an increasing protein concentration applied. The latter implies that the Fn density on the gelatin films can be easily finetuned by varying the Fn concentration applied during coating (e.g. by dip coating). Figure 6B further indicates that within the applied concentration range a linear correlation $\left(\mathrm{r}^{2}=0.95\right)$ exists between the applied Fn concentration and the SPR response.

It is anticipated that the orientation of the attached Fn molecule determines possible effects on cell anchorage, since the cell attachment mechanism is thought to involve the linkage of $\alpha_{5} \beta_{1}$ 
transmembrane integrin receptors to the Fn cell binding site. ${ }^{[24]}$ A frequently applied test for binding site availability which also gives an estimation of the molecular orientation, is the degree to which antibodies bind to immobilized proteins. ${ }^{[25]}$ Therefore, a Fn-antibody was flushed over the Fn-coated gelatin surface. Interestingly, the response of the anti-Fn did not increase with the amount of Fn adsorbed on the surface (see figure 6A). It can be anticipated that Fn multilayers were deposited onto the sensor chip, while the anti-Fn can only interact with the top Fn layer.

Gelatin B is processed by an alkaline treatment. ${ }^{[26]}$ The alkaline pre-treatment converts glutamine and aspargine residues into glutamic acid and aspartic acid, which results in a carboxylic acid content of 118 per 1000 amino acids for gelatin B. At physiological pH, the gelatin $\mathrm{B}$ chains possess a net negative charge due to the presence of $-\mathrm{COO}^{-}$groups.

Since type B gelatin and Fn possess iso-electric points in the range of 5-6, both biopolymers are negatively charged at physiological $\mathrm{pH}^{[26,27]}$ This implies that gelatin type $\mathrm{B} / \mathrm{Fn}$ interactions are mainly hydrophobic in nature. A second type of gelatin which has been applied for the development of biomaterials, is gelatin type A, obtained after an acidic treatment. ${ }^{[28]}$ Gelatin type A possesses an iso-electric point of 8 and is positively charged at physiological $\mathrm{pH}$. It can be anticipated that in addition to hydrophobic interactions, electrostatic interactions with Fn can also occur.

In order to study the possible effects of the iso-electric point on the Fn affinity, gel-MOD (type A) was also spincoated on an SPR sensor and crosslinked subsequently. Again, experiments were performed confirming the presence and the stability of the spincoated gelMOD layer on the SPR chip (data not shown). The gel-MOD (type A) coated SPR chips were subsequently used for studying the interaction with Fn (1-200 $\mu \mathrm{g} / \mathrm{ml})$. The sensorgrams (see figure 6C) indicate that the amount of adsorbed Fn again increased with an increasing Fn 
concentration applied. In similarity to gelatin type $B$, a linear correlation $\left(r^{2}=0.95\right)$ between the Fn concentration and the SPR response was obtained (figure 6D).

\subsubsection{Study of binding kinetics}

In all SPR experiments described in the previous paragraph, a flow rate of $50 \mu 1 / \mathrm{min}$ was applied in order to exclude mass transport limitations, thus enabling evaluation of the binding kinetics. ${ }^{[29]}$ Table 3 shows the association and dissociation constants characterizing the Fn affinity for both gelatin type A and type B. The association constant obtained for type B gelatin (i.e. $5.31 \times 10^{8} \mathrm{M}^{-1}$ ) corresponds to values reported earlier for the association of $\mathrm{Fn}$ with a Fn-binding peptide sequence in gelatin (i.e. $2 \times 10^{8} \mathrm{M}^{-1}$ ) as well as denatured collagen (i.e. $\left.4 \times 10^{8} \mathrm{M}^{-1}\right) \cdot{ }^{[30,31]}$ As anticipated, the association constant for the interaction with type A gelatin was higher compared to type B gelatin $\left(1.24 \times 10^{10} \mathrm{M}^{-1}\right.$ versus $\left.5.31 \times 10^{8} \mathrm{M}^{-1}\right)$.

\subsection{Quartz Crystal Microbalance experiments}

In contrast to SPR, QCM is a technique which enables quantitative analysis of the amount of adsorbed Fn on the gelatin surface. In order to enable a quantification of the amount of Fn coated on the gelatin films, we therefore performed a series of QCM experiments. QCM enables monitoring various properties of the deposited layer on the crystal surface including mass, thickness and visco-elasticity.

QCM crystals were spincoated using an aqueous gel-MOD solution (5 w/v\%, type A and B). In order to verify the presence and the stability of modified gelatin onto the crystal surface, ATR-IR spectroscopy, contact angle measurements and atomic force microscopy were performed (data not shown). The gelatin (type A and B) mass deposited on the QCM chips was obtained by measuring the frequency differences between both an uncoated and a gelMOD-coated crystal in air using QCM. All frequency values (overtones etc.) were in 
excellent agreement (data not shown) enabling mass determination using the Sauerbrey relation:

$$
\Delta \mathrm{m}=-\frac{C \quad f}{n}
$$

$C=17.7 \mathrm{ng} \cdot \mathrm{Hz}^{-1} \cdot \mathrm{cm}^{-2}$ for a $5 \mathrm{MHz}$ crystal; $\mathrm{n}=$ the overtone number; $\Delta \mathrm{f}=$ the frequency shift Spincoating of type A and type B gel-MOD resulted in mass depositions of respectively 18 $\mu \mathrm{g} / \mathrm{cm}^{2}$ and $16 \mu \mathrm{g} / \mathrm{cm}^{2}$ on the QCM crystals.

The gel-MOD-coated crystals obtained were subsequently used for studying the interaction with Fn at concentrations of 1 and $25 \mu \mathrm{g} / \mathrm{ml}$.

If the adsorbed layer is not rigid, the Sauerbrey relation becomes invalid. Because a viscoelastic film will not fully couple to the oscillation of the crystal, the Sauerbrey equation underestimates the mass deposited at the surface. ${ }^{[32]}$

A viscoelastic film dampens the crystal's oscillation. The dissipation of the oscillation of the quartz crystal gives an idea on the viscoelastic properties of the attached layer. The dissipation is determined according to the following equation:

$$
\mathrm{D}=\frac{E_{\text {lost }}}{2 \pi E_{\text {stored }}}
$$

$\mathrm{E}_{\text {lost }}=$ energy dissipated during one oscillation cycle; $\mathrm{E}_{\text {stored }}=$ energy stored in the oscillator The dissipation of a crystal can be measured by recording the response of a freely oscillating crystal, vibrating at its resonance frequency. By measuring at multiple frequencies and applying the Voight model, the deposited viscoelastic film can be characterized in detail. Sauerbrey as well as Voight masses were calculated respectively using equation (3) and (4). Different frequency and dissipation responses indicated visco-elastic behaviour of the proteins deposited on the crystal surface. As a result, only Voight masses were taken into account and compared. An overview of film thicknesses and Voight masses are summarized in table 4. The data obtained for the masses include the amount of water deposited on the sensor crystal. 
The data indicate that the deposited mass increases when increasing the applied Fn concentration. In accordance to the SPR experiments, the QCM data indicate a higher affinity of gelatin type A for Fn compared to type B. From the measured layer thickness values, it can be seen that in some cases, the values obtained are lower than the size of Fn itself (i.e. $16 \mathrm{~nm}$ as obtained using dynamic light scattering measurements). This can be attributed to the fact that the QCM results obtained, are an average for the entire chip. When the chip is not completely coated, QCM underestimates the overall thickness. In addition, we anticipate that Fn denaturation occurs to some extent since one of the driving forces for the gelatin-Fn affinity is hydrophobic in nature. The latter phenomenon also results in lower Fn layer thicknesses on gelatin-coated QCM chips.

\subsection{Radiolabelling experiments}

Since QCM and SPR measurements are limited to monitoring interactions at surfaces, an alternative technique was elaborated enabling the study of the Fn affinity for 3D porous gelatin hydrogels.

In a first part, $10 \mathrm{w} / \mathrm{v} \%$ crosslinked gel-MOD (type A and type B) hydrogel films were prepared and their Fn affinity was evaluated. This enabled correlation with the SPR and the QCM data obtained. The hydrogel films prepared ( $66 \mathrm{~mm}$ x $1 \mathrm{~mm})$ were incubated for 1 hour in PBS at ambient temperature, in the presence of ${ }^{125}$ I radiolabelled Fn $(1-200 \mu \mathrm{g} / \mathrm{ml})$. The polymer films were washed three times in PBS, prior to radioactivity measurements. The experiments were performed in triplicate and the mean values are depicted in figure 7.

In figure 7, the Fn mass adsorbed by the hydrogel films is plotted as a function of the applied concentration. The results confirm that type A gel-MOD possesses a higher affinity for Fn compared to type B gel-MOD. These data are in agreement with the SPR and QCM data obtained. The plot of the adsorbed mass of Fn as a function of the Fn concentration flattened 
off at elevated concentrations. This effect was due to saturation of the surface, as demonstrated in figure 7.

In table 5, both QCM and radiolabelling results are depicted. The mass of bound Fn is shown as a function of the gelatin type and the Fn concentration. SPR results are not included since SPR is a qualitative technique rather than a quantitative method. Table 5 indicates that the obtained results differ according to the technique applied. A first parameter influencing the results, is that the conformation of spincoated gel-MOD (cfr. QCM) differs from that of gelMOD, present in hydrogel films (cfr. radiolabelling). In addition, the deposited mass of Fn onto the QCM crystals, is obtained using an estimated density of $1050 \mathrm{~kg} / \mathrm{m}^{3}$ for the Fn layer.

In a last part of the work, a series of tests were performed to study to what extent the interaction between Fn and porous 3D scaffolds could be determined. Two different types of pore size/geometry were included. Type I scaffolds consisted of cone-shaped pores, with a pore size decreasing from top to bottom (330 to $20 \mu \mathrm{m}$ ) and type II hydrogels were composed of spherical pores, possessing a diameter of $135 \mu \mathrm{m}$ (table 2, figure 8). Both type A and type B gel-MOD were applied to obtain hydrogels possessing respectively a positive and a negative overall charge.

Scaffolds with varying pore size and pore geometry were prepared as reported earlier (see table 2). ${ }^{[12]}$ The materials developed were compared in terms of their Fn affinity. This is of relevance since it is anticipated that Fn uptake in the gelatin cryogels developed would improve their in vitro biocompatibility. In addition to the influence of the pore size and the pore geometry, the effect of the gelatin charge (cfr. iso-electric point) on the Fn affinity was also evaluated. The four Gel-MOD combinations obtained (type AI, type AII, type BI and type BII) scaffolds were incubated in aqueous ${ }^{125} \mathrm{I}-\mathrm{Fn}$ solutions $(1-200 \mu \mathrm{g} / \mathrm{ml})$ for $1 \mathrm{~h}$ at 
ambient temperature to monitor their Fn interaction. In figure 9, the absorbed Fn mass is depicted as a function of both the Fn concentration and the scaffold type applied. The results indicate that type I scaffolds interacted to a higher extent with Fn compared to type II hydrogels (figure 9) for both type A and type B gelatin. This phenomenon is most likely related to differences in mass transport phenomena between type I and type II hydrogels. The latter assumption is supported by the different water uptake capacity of type I and type II hydrogels (see figure 10). The channel-like pore morphology of type I hydrogels leads to a higher swelling compared to the one observed in type II hydrogels (figure 10 ). ${ }^{[33]}$ It should be mentioned that both hydrogel types are fully interconnected, ${ }^{[34]}$ which implies that pore interconnectivity can be ruled out as a factor determining the Fn adsorption.

In addition to the effect of the structural properties, the influence of the overall charge of the gelatin hydrogels on the Fn affinity was also evaluated. Figure 9 indicates that gel-MOD A based scaffolds adsorbed/absorbed less Fn compared to gel-MOD B based matrices. The latter observation was not anticipated since, in addition to hydrophobic interactions (in case of type B gelatin and Fn), type A gelatin and Fn also interact through electrostatic interactions. Therefore we determined the water uptake capacity of both gelatin A and gelatin B based hydrogels. The results (see figure 10) indicated that the water uptake capacity of type A gelMOD scaffolds was lower compared to the water uptake capacity of type B gel-MOD hydrogels. The latter is due to electrostatic repulsions caused by the carboxylate groups of gelatin B (see paragraph 3.1.1) and thus resulted in larger scaffold expansion compared to type A gelatin. This phenomenon leads to a higher water uptake capacity of type B hydrogels. The latter observation is in agreement with earlier literature reports. ${ }^{[35-38]}$ As examples: Bajpai et al investigated the effect of the gelatin type on the release profile of sulphamethoxazole by loading the drug on both gelatin A and B nanoparticles. The results indicated that the fractional drug release from type B gelatin was higher compared to type A gelatin. The results 
were ascribed to the larger swelling capacity of type B nanoparticles. ${ }^{[35]}$ Similar results were obtained for the release of chloroquine phosphate from gelatin nanoparticles. ${ }^{[38]}$ As a result, type A scaffolds possess a lower Fn affinity compared to type B derivatives. 


\section{Conclusion}

Various techniques including surface plasmon resonance, quartz crystal microbalance and radiolabelling experiments were applied to study the interaction between Fn and gelatin. A detailed study of the interactions occurring is of great relevance in the field of biomaterials science since Fn is often applied as top layer in the design of implant materials.

The present work indicates that gelatin-based hydrogels can be coated using cell-interactive proteins including Fn. The amount of incorporated Fn can be easily finetuned by varying the applied concentration. Type A gelatin films showed a higher affinity for fibronectin compared to type B gelatin films, as anticipated based on their iso-electric points. Porous gelatin scaffolds, however, showed an opposite trend. Type B scaffolds incorporated a higher Fn amount compared to type A matrices due to larger expansion possibilities of type B scaffolds. In addition to the gelatin charge, the Fn affinity is also influenced by structural properties of the hydrogel scaffolds including the pore size and the pore geometry. 


\section{Acknowledgements}

The authors would like to acknowledge the Research Foundation - Flanders (FWO) and the Institute for the Promotion of Innovation by Science and Technology in Flanders (IWT) for the research grants assigned to S. Van Vlierberghe and E. Vanderleyden. The authors would also like to thank the PolExGene consortium. PolExGene is a STREP project (contract number 019114) funded under the EU 6th framework programme. 


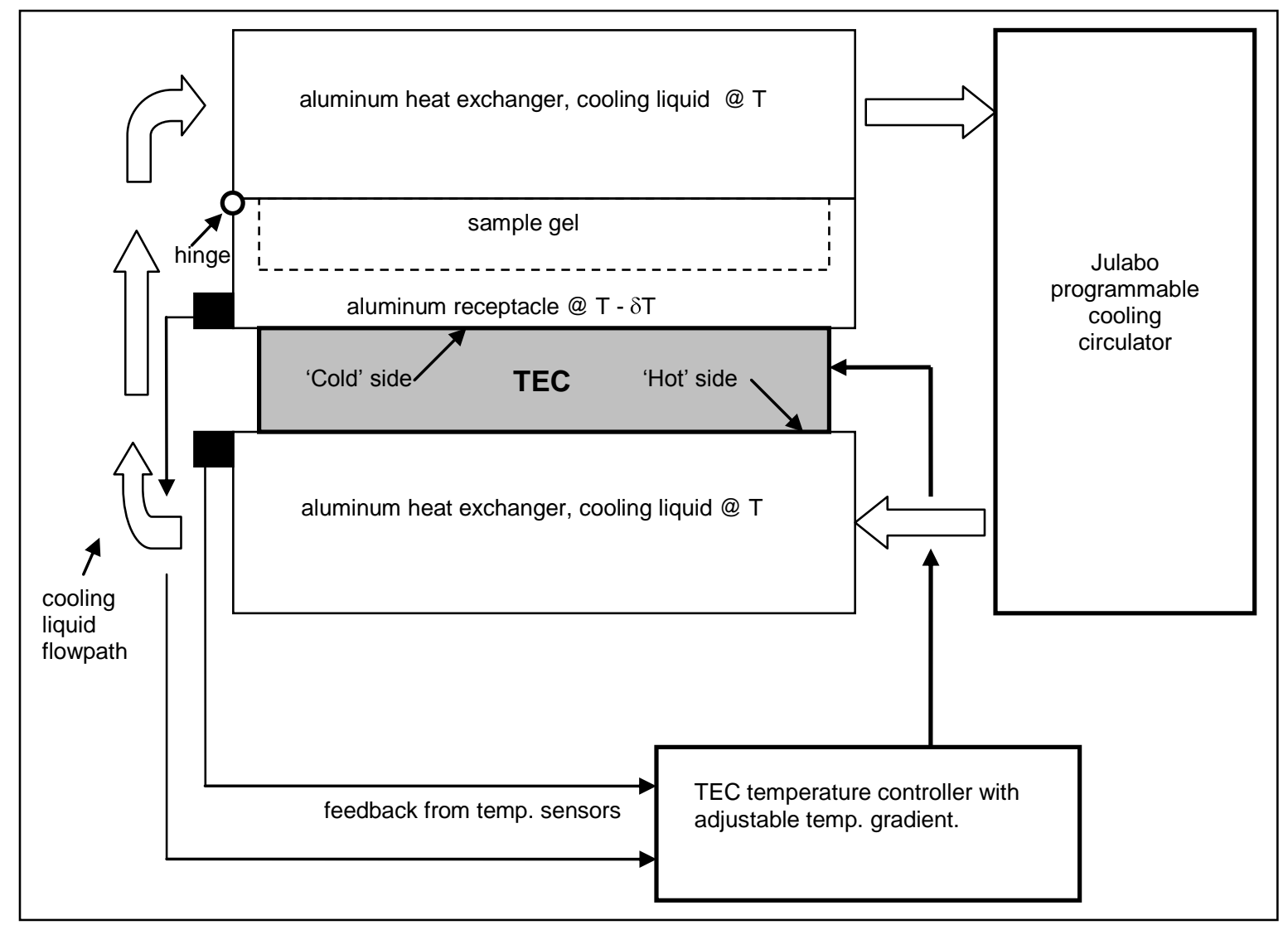

Figure 1 


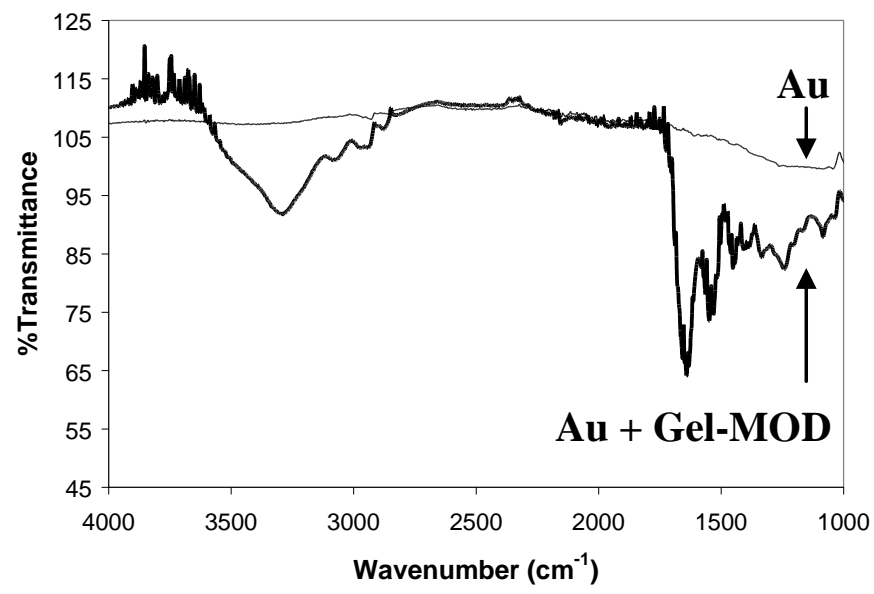

Figure 2 


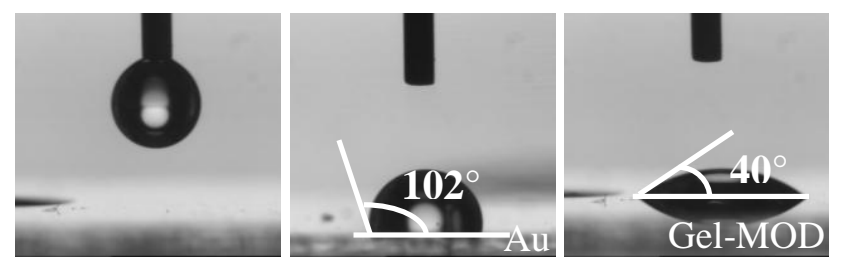

Figure 3 
A

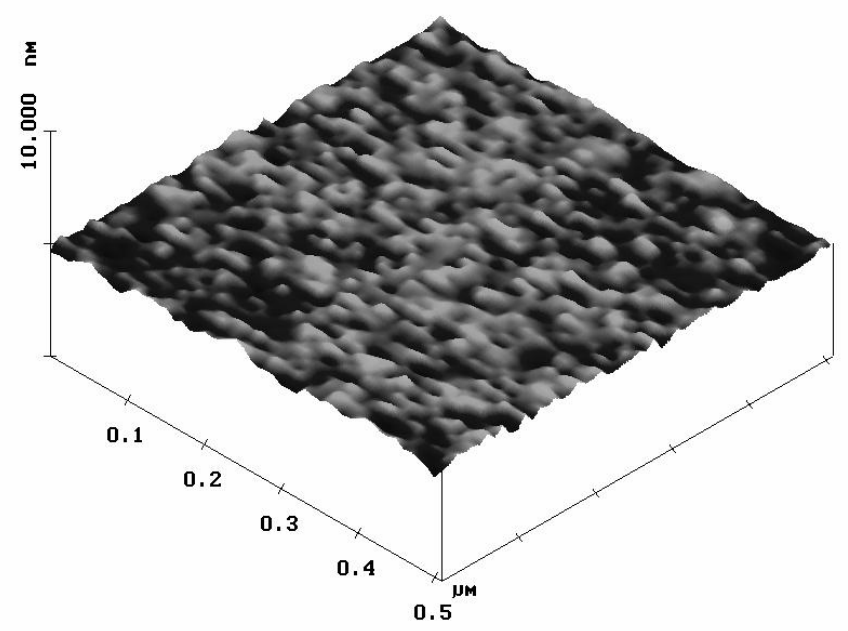

B

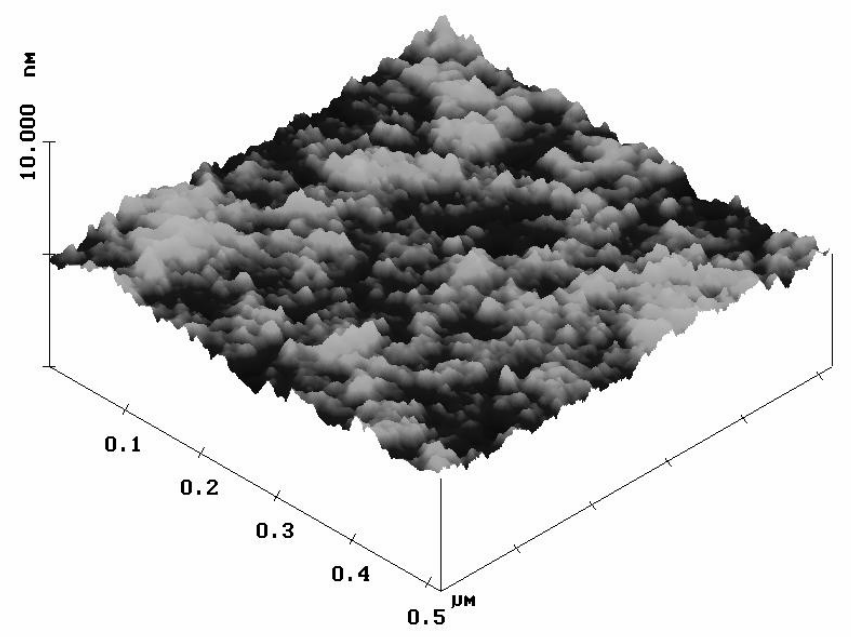

Figure 4 


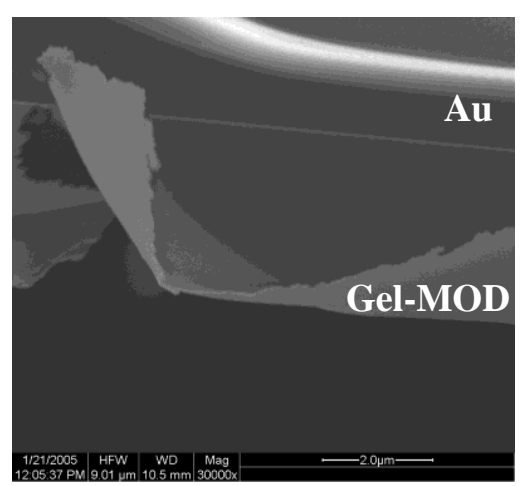

Figure 5 
A

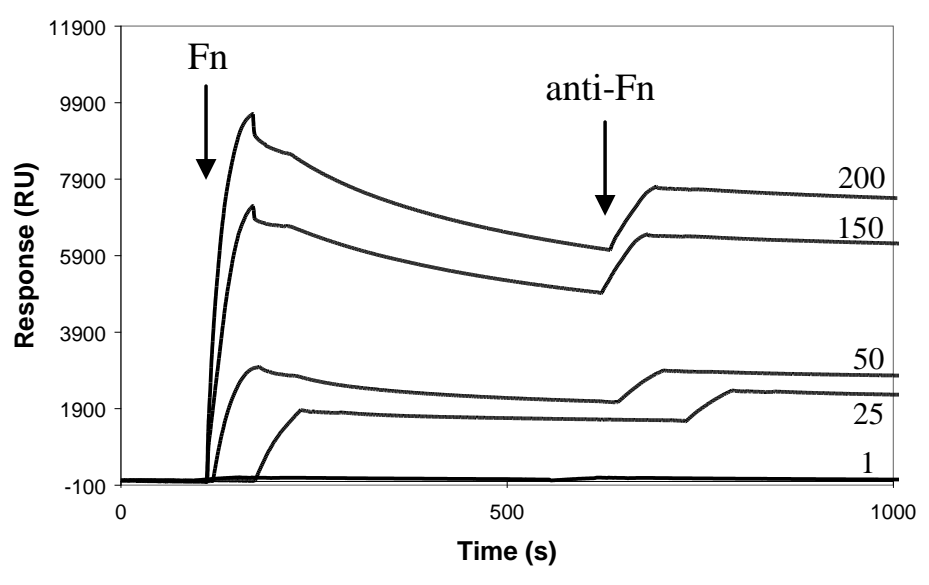

B

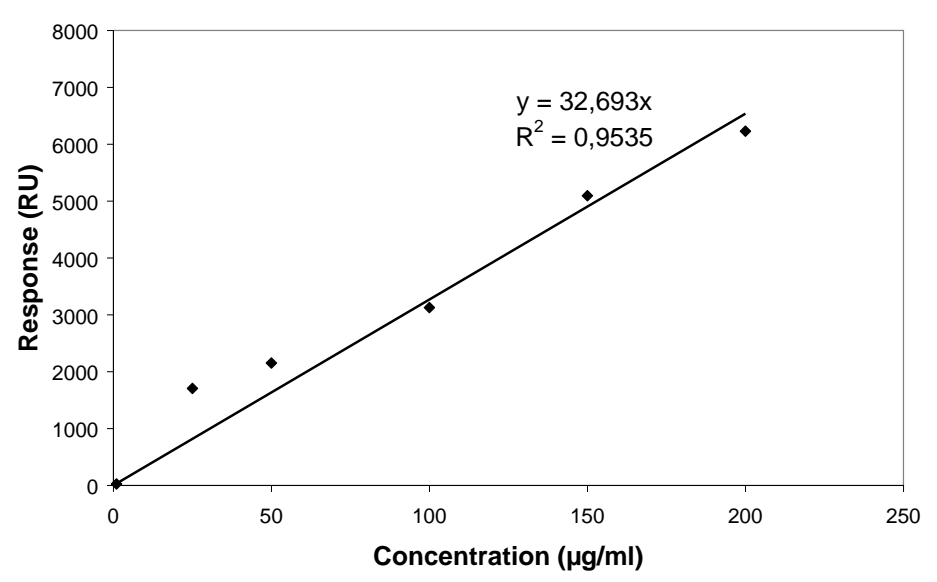

C

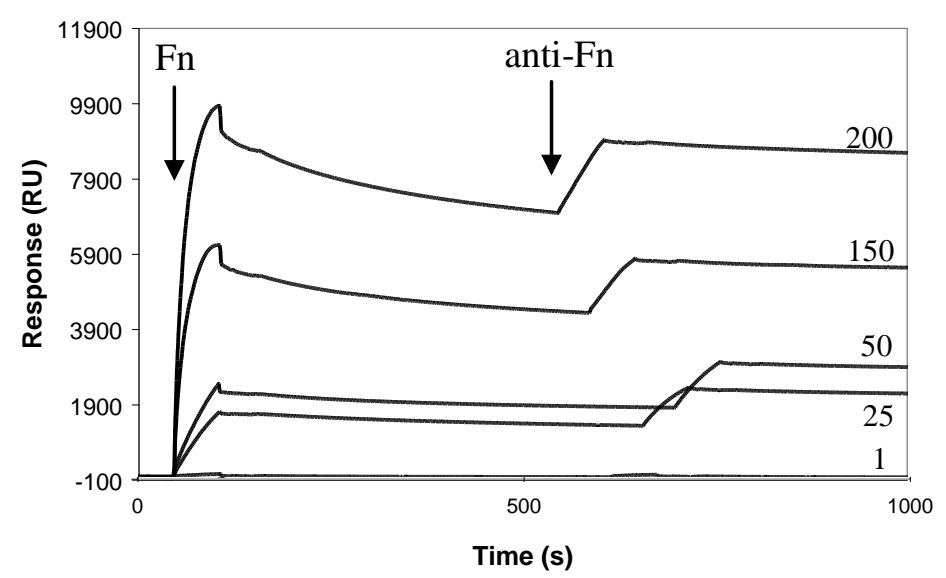

D

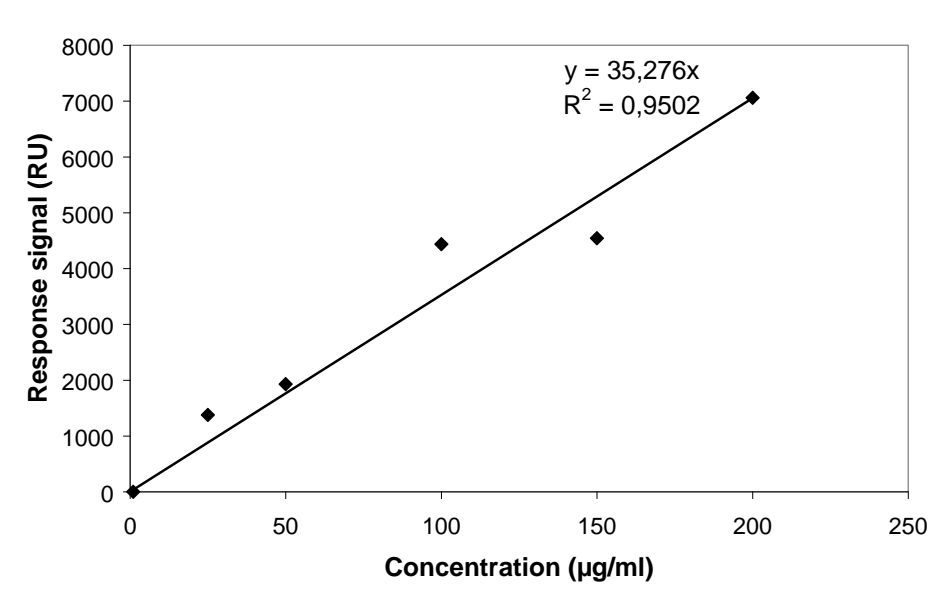

Figure 6 
- Type A - Type B

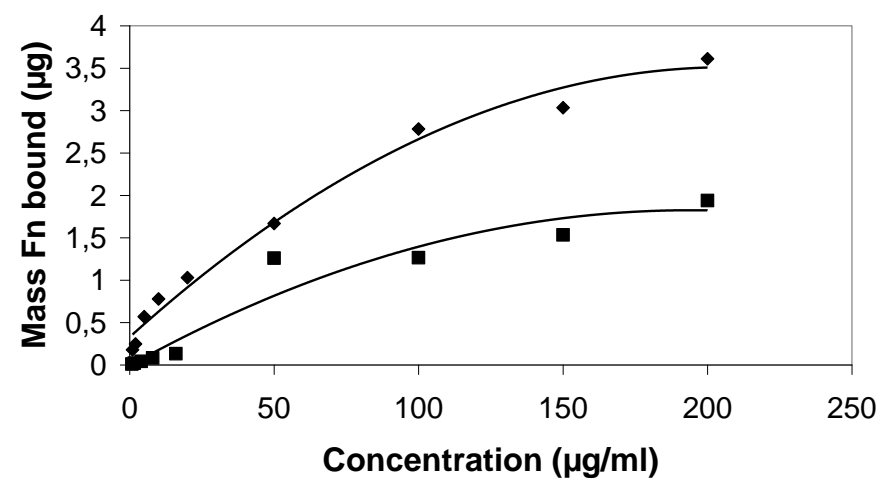

Figure 7 
A

Type I

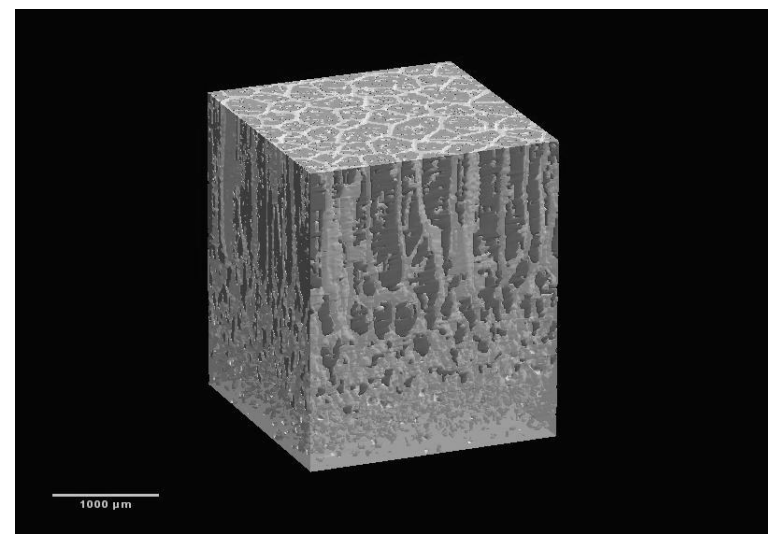

C

Type I, top view

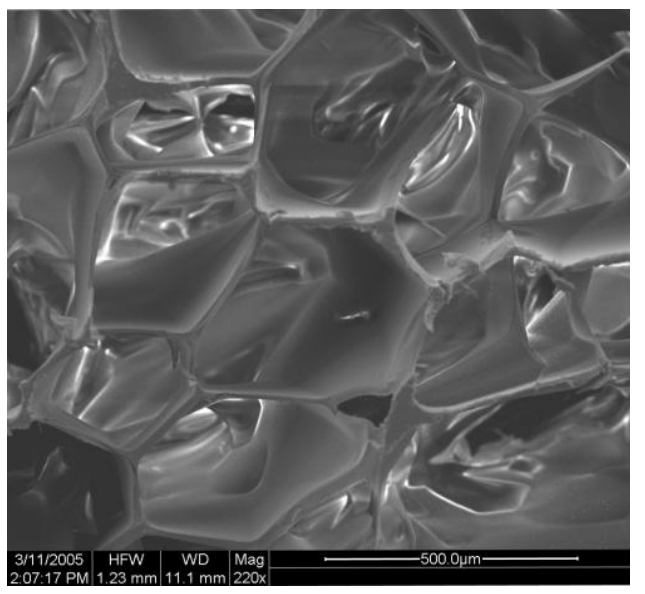

B

Type II

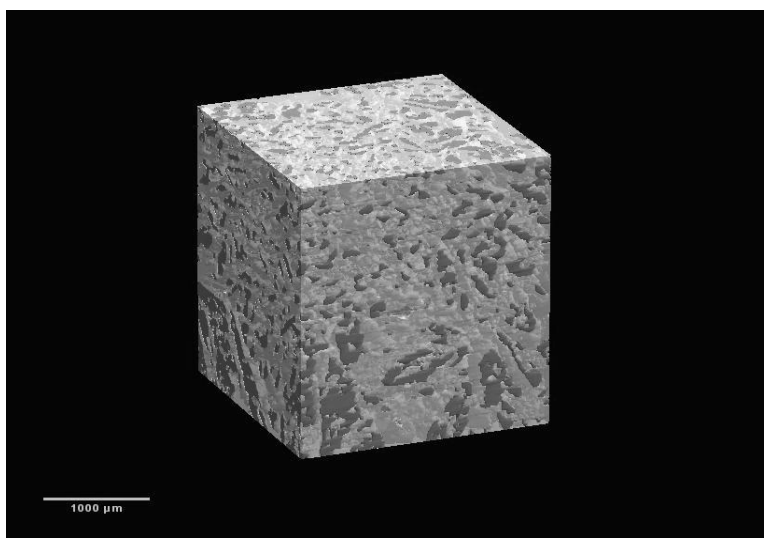

D

Type II, top view

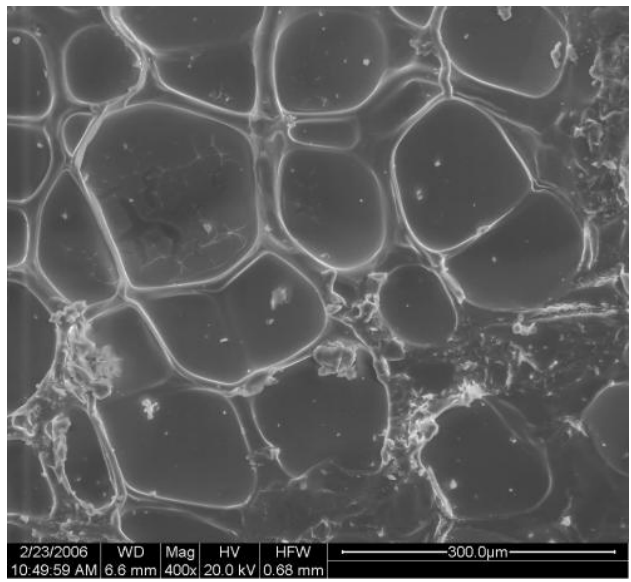

Figure 8 


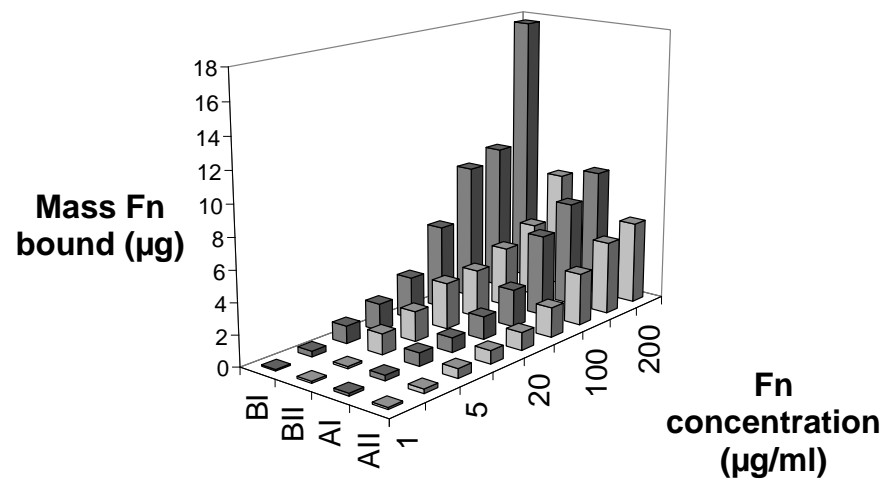

Figure 9 


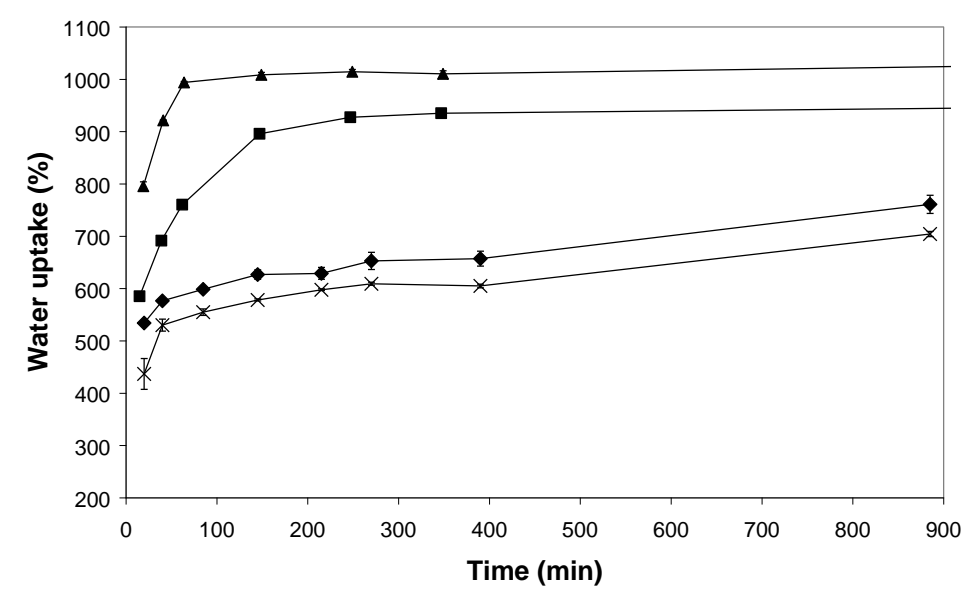

Figure 10 
Figure 1. Schematic overview of the programmable cryo-unit, applied for the preparation of the porous gelatin scaffolds. Reprinted by permission from American Chemical Society: [Biomacromolecules] (Van Vlierberghe S et al., Porous Gelatin Hydrogels: 1. Cryogenic Formation and Structure Analysis, Vol 8, p. 331-337). Copyright (2007).

Figure 2. ATR-IR-spectra of a non-coated sensor chip (Au) and a gel-MOD (5 w/v\%, type B) spincoated sensorchip ( $\mathrm{Au}+\mathrm{Gel-MOD}$, type $\mathrm{B}$ ) at $25^{\circ} \mathrm{C}$.

Figure 3. Contact angle measurements on gold and on a sensor chip, coated with gel$\operatorname{MOD}(5 \mathrm{w} / \mathrm{v} \%$, type B).

Figure 4. Surface plots $(0.5 \times 0.5 \mu \mathrm{m} \times 10 \mathrm{~nm})$ of (A) an uncoated SPR chip and (B) a gel-MOD (type B) coated chip obtained using AFM.

Figure 5. SEM image of a cross-section of a gold chip, after spincoating with gel-MOD ( $5 \mathrm{w} / \mathrm{v} \%$, type B). The scale bar represents $2 \mu \mathrm{m}$.

Figure 6. Part A: Sensorgrams showing the SPR response upon rinsing aqueous Fn solutions (1 - $200 \mu \mathrm{g} / \mathrm{ml}$ ) over a gel-MOD (type B) coated chip, followed by an aqueous anti-Fn solution $(0.05 \mathrm{mg} / \mathrm{ml})$.

Part B: SPR response of gel-MOD (type B) as a function of the Fn concentration applied.

Part C: Sensorgrams showing the SPR response upon rinsing aqueous Fn solutions (1 - $200 \mu \mathrm{g} / \mathrm{ml})$ over a gel-MOD (type A) coated chip, followed by an aqueous anti-Fn solution $(0.05 \mathrm{mg} / \mathrm{ml})$.

Part D: SPR response of gel-MOD (type A) as a function of the Fn concentration applied.

Figure 7. Adsorbed Fn amount $(\mu \mathrm{g})$ as a function of the Fn concentration and the hydrogel type applied as measured by radiolabelling experiments. 
Figure 8. Micro-CT and SEM analysis of type I and type II gelatin hydrogels. Row 1-2 represent micro-CT and SEM data respectively of both types of porous hydrogels. For the micro-CT pictures (part A-B), the pores are coloured dark grey and the scaffold material is light grey. The scale bars represent $1000 \mu \mathrm{m}$ (part A-B), $500 \mu \mathrm{m}$ (part C), $300 \mu \mathrm{m}$ (part D). Reprinted with permission from American Chemical Society: [Biomacromolecules] (Dubruel P et al., Porous Gelatin Hydrogels: 2. In Vitro Cell Interaction Study, Vol 8, p. 338-344). Copyright (2007).

Figure 9. Absorbed Fn amount $(\mu \mathrm{g})$ as a function of the Fn concentration and the scaffold type applied as determined using radiolabelling experiments.

Figure 10. Water uptake capacity as a function of time for $10 \mathrm{w} / \mathrm{v} \%$ hydrogels: gel-MOD B, type I (-); gel-MOD B, type II (-); gel-MOD A, type I ( $)$; gel-MOD A, type II (*). 
Table 1. Advantages and disadvantages of surface plasmon resonance, quartz crystal microbalance and radiolabelling experiments.

\begin{tabular}{|c|c|c|}
\hline & Advantages & Disadvantages \\
\hline $\begin{array}{c}\text { Surface plasmon } \\
\text { resonance }\end{array}$ & $\begin{array}{l}\text { very sensitive }\left(10^{-11} \mathrm{M}\right) \\
\text { label-free } \\
\text { real-time monitoring } \\
\text { qualitative } \\
\text { information on kinetics } \\
\text { easy sample handling } \\
\text { fast }\end{array}$ & $\begin{array}{l}\text { lower sensitivity than fluorescence } \\
\text { heterogeneities averaged out } \\
\text { curve fitting by means of models } \\
\text { no 3D analysis }\end{array}$ \\
\hline $\begin{array}{l}\text { Quartz crystal } \\
\text { microbalance }\end{array}$ & $\begin{array}{l}\text { very sensitive }\left(0.5 \mathrm{ng} . \mathrm{cm}^{-2}\right) \\
\text { label-free } \\
\text { real-time monitoring } \\
\text { quantitative } \\
\text { mechanical properties } \\
\text { simple setup and use } \\
\text { fast }\end{array}$ & $\begin{array}{l}\text { lower sensitivity than fluorescence } \\
\text { based on modelling } \\
\text { no 3D analysis }\end{array}$ \\
\hline Radiolabelling & $\begin{array}{l}\text { quantitative } \\
\text { 3D analysis }\end{array}$ & $\begin{array}{l}\text { time consuming } \\
{ }^{125} \text { I radioactivity hazard }\end{array}$ \\
\hline
\end{tabular}


Table 2. Summary of the cryogenic parameters applied and hydrogel characteristics (pore size, pore morphology and porosity) of type I and type II, $10 \mathrm{w} / \mathrm{v} \%$ gelMOD hydrogels as studied by $\mu$-CT (pore size and morphology) and Hepycnometry (porosity).

\begin{tabular}{|c|c|c|}
\hline \multirow{2}{*}{} & \multicolumn{2}{|c|}{ Hydrogel Type } \\
\cline { 2 - 3 } & I & II \\
\hline Cooling range & $21{ }^{\circ} \mathrm{C}$ to $-30^{\circ} \mathrm{C}$ & $21{ }^{\circ} \mathrm{C}$ to $-30^{\circ} \mathrm{C}$ \\
\hline Cooling rate & $0.15^{\circ} \mathrm{C} / \mathrm{min}$ & $0.15^{\circ} \mathrm{C} / \mathrm{min}$ \\
\hline Temperature gradient & $30^{\circ} \mathrm{C}$ & none \\
\hline Pore morphology & $\begin{array}{c}\text { cone, pore size } \\
\text { decreasing from } \\
\text { top to bottom }\end{array}$ & $\begin{array}{c}\text { spherical, uniform pore } \\
\text { size throughout entire } \\
\text { scaffold }\end{array}$ \\
\hline $\begin{array}{c}330 \rightarrow 20 \mu \mathrm{m} \\
\text { Pore size } \\
\text { (Porosity) }\end{array}$ & $\begin{array}{c}135 \mu \mathrm{m} \\
\text { top } \\
(94 \%)\end{array}$ \\
\hline \multicolumn{2}{|c|}{} \\
\hline
\end{tabular}


Table 3. Association and dissociation constants obtained using SPR.

\begin{tabular}{|c|ccc|}
\hline & $\mathbf{k}_{\mathbf{a}}\left(\mathbf{M}^{\mathbf{- 1}} \mathbf{s}^{\mathbf{- 1}}\right)$ & $\mathbf{k}_{\mathbf{d}}\left(\mathbf{s}^{\mathbf{- 1}}\right)$ & $\mathbf{K}_{\mathbf{A}}\left(\mathbf{M}^{\mathbf{- 1}}\right)$ \\
\hline Type B & $2.76 \times 10^{5}$ & $5.21 \times 10^{-4}$ & $5.31 \times 10^{8}$ \\
\hline Type A & 252 & $2.03 \times 10^{-8}$ & $1.24 \times 10^{10}$ \\
\hline
\end{tabular}


Table 4. Overview showing the QCM results, obtained by rinsing Fn (1 and $25 \mu \mathrm{g} / \mathrm{ml})$ and anti-Fn $(50 \mu \mathrm{g} / \mathrm{ml})$ over sensor crystals, after spincoating with respectively type A and type B gel-MOD.

\begin{tabular}{|cc|cc|cc|}
\hline & \multicolumn{2}{|c|}{ Type A gelatin } & \multicolumn{2}{c|}{ Type B gelatin } \\
& & $1 \mu \mathrm{g} / \mathrm{ml}$ & $25 \mu \mathrm{g} / \mathrm{ml}$ & $1 \mu \mathrm{g} / \mathrm{ml}$ & $25 \mu \mathrm{g} / \mathrm{ml}$ \\
\hline \multirow{2}{*}{ Fn } & thickness $(\mathrm{nm})$ & 0.7 & 18 & 0.3 & 1 \\
& mass $\left(\mathrm{ng} / \mathrm{cm}^{2}\right)$ & 73 & 1837 & 31 & 105 \\
\hline \multirow{2}{*}{ anti-Fn } & thickness $\left(\mathrm{nm}^{2}\right)$ & 3 & 22 & 1.2 & 1.4 \\
& mass $\left(\mathrm{ng} / \mathrm{cm}^{2}\right)$ & 241 & 472 & 126 & 147 \\
\hline
\end{tabular}


Table 5. Comparison of results obtained using QCM and radiolabelling experiments on gel-MOD type A and type B.

\begin{tabular}{|cc|cc|cc|}
\hline & \multicolumn{2}{|c|}{ Type A gelatin } & \multicolumn{2}{c|}{ Type B gelatin } \\
& & $1 \mu \mathrm{g} / \mathrm{ml}$ & $25 \mu \mathrm{g} / \mathrm{ml}$ & $1 \mu \mathrm{g} / \mathrm{ml}$ & $25 \mu \mathrm{g} / \mathrm{ml}$ \\
\hline Mass & $Q C M$ & 73 & 1837 & 31 & 105 \\
$\left(\mathbf{n g} / \mathbf{c m}^{\mathbf{2}}\right)$ & Radiolabelling & 239 & 1636 & 15 & 739 \\
\hline
\end{tabular}




\section{References}

[1] H. J. Kim, I. T. Chang, S. J. Heo, J. Y. Koak, S. K. Kim, J. H. Jang, Clinical Oral Implants Research 2005, 16, 557.

[2]

[3] G. Altankov, V. Thom, T. Groth, K. Jankova, G. Jonsson, M. Ulbricht, Journal of Biomedical Materials Research 2000, 52, 219.

[4] S. Badylak, A. Liang, R. Record, R. Tullius, J. Hodde, Biomaterials 1999, 20, 2257.

[5] E. Detrait, J. B. Lhoest, P. Bertrand, P. V. de Aguilar, Journal of Biomedical Materials Research 1999, 45, 404.

[6] J. Hodde, R. Record, R. Tullius, S. Badylak, Biomaterials 2002, 23, 1841.

[7] A. J. Bergman, K. Zygourakis, Biomaterials 1999, 20, 2235.

[8] S. P. Palecek, J. C. Loftus, M. H. Ginsberg, D. A. Lauffenburger, A. F. Horwitz, Nature 1997, 385, 537.

[9] Y. Z. Yang, R. Glover, J. L. Ong, Colloids and Surfaces B-Biointerfaces 2003, 30, 291.

[10] Y. Z. Yang, R. Cavin, J. L. Ong, Journal of Biomedical Materials Research Part A 2003, $67 A, 344$

[11] D. W. Grainger, G. Pavon-Djavid, V. Migonney, M. Josefowicz, Journal of Biomaterials Science-Polymer Edition 2003, 14, 973.

[12] S. VanVlierberghe, V. Cnudde, P. Dubruel, B. Masschaele, A. Cosijns, I. DePaepe, P. J. S. Jacobs, L. VanHoorebeke, J. P. Remon, ～E. Schacht, Biomacromolecules 2007, 8, 331.

[13] P. Dubruel, R. Unger, S. VanVlierberghe, V. Cnudde, P. J. S. Jacobs, E. Schacht, C. J. Kirkpatrick, Biomacromolecules 2007, 8, 338.

[14] E. Engvall, $\quad$ E. Ruoslahti, International Journal of Cancer 1977, $20,1$.

[15] K. C. Ingham, S. A. Brew, S. I. Miekka, Molecular Immunology 1983, 20, 287. 
[16] P. Speziale, L. Visai, S. Rindi, A. Di Poto, Nature Protocols 2008, 3, 525.

[17] G. R. Alvarez, I. M. Cabrera, A. G. Rodriguez, R. B. Maura, A. M. Torres, M. D. A. P. Collazo, M. A. Sanchez, R. C. Arias, L. G. Imias, Latin American Journal of Pharmacy 2007, 26, 165.

[18] S. I. Miekka, K. C. Ingham, ～D. Menache, Thrombosis Research 1982, 27, 1.

[19] M. Vuento, U. H. Stenman, E. Salonen, K. Osterlund, P. Kuusela, Molecular Immunology 1983, 20, 149.

[20] L. I. Gold, ～E. Pearlstein, Biochimica Et Biophysica Acta 1979, 581, 237.

[21] Technical data sheet, DakoCytomation

[22] A. I. Van den Bulcke, B. Bogdanov, N. De Rooze, E. H. Schacht, M. Cornelissen, H. Berghmans, Biomacromolecules 2000, 1, 31.

[23] I. Burvenich, S. Schoonooghe, B. Cornelissen, P. Blanckaert, E. Coene, C. Cuvelier, N. Mertens, G. Slegers, Clin Cancer Res 2005, 11, 7288.

[24] C. R. Wittmer, J. A. Phelps, W. M. Saltzman, P. R. Van Tassel, Biomaterials 2007, 28, 851.

[25] C. R. Wittmer, P. R. Van Tassel, Colloids and Surfaces B-Biointerfaces 2005, 41, 103.

[27] K. B. Djagny, Z. Wang, S. Y. Xu, Critical Reviews in Food Science and Nutrition $2001,41,481$.

[28]

[29] J. Svitel, H. Boukari, D. Van Ryk, R. C. Willson, P. Schuck, Biophysical Journal 2007, $92,1742$.

[30] X. Y. Gao, M. J. Groves, European Journal of Pharmaceutics and Biopharmaceutics $1998,45,275$.

[31] J. S. Tjia, B. J. Aneskievich, P. V. Moghe, Biomaterials 1999, 20, 2223. 
[32] M. S. Lord, C. Modin, M. Foss, M. Duch, A. Simmons, F. S. Pedersen, B. K. Milthorpe, F. Besenbacher, Biomaterials 2006, 27, 4529.

[33] S. Van Vlierberghe, P. Dubruel, E. Lippens, M. Cornelissen, E. Schacht, Journal of Biomaterials Science-Polymer Edition 2009, accepted,

[34] S. Van Vlierberghe, V. Cnudde, P. Dubruel, B. Masschaele, A. Cosijns, I. De Paepe, P. J. S. Jacobs, L. Van Hoorebeke, J. P. Remon, $\quad$ E. Schacht, Biomacromolecules 2007, 8, 331.

[35] A. K. Bajpai, J. Choubey, Journal of Macromolecular Science-Pure and Applied Chemistry 2005, A42, 253.

[36] A. J. Kuijpers, G. H. M. Engbers, P. B. van Wachem, J. Krijgsveld, S. A. J. Zaat, J. Dankert, J. Feijen, Journal of Controlled Release 1998, 53, 235.

[37] M. Bessho, M. Furuta, T. Kojima, S. Okuda, M. Hara, Journal of Biomaterials Science-Polymer Edition 2005, 16, 715.

[38] A. K. Bajpai, C. Jyoti, Journal of Materials Science: Materials in Medicine 2006, 17, 345. 


\section{Table of contents}

The affinity of gelatin type A and B for fibronectin was evaluated using surface plasmon resonance, quartz crystal microbalance and radiolabelling experiments. The affinity study was performed on both hydrogel films and 3D scaffolds. In addition to quantifying the amount of adsorbed/absorbed Fn, the binding kinetics of Fn and gelatin were evaluated in depth.

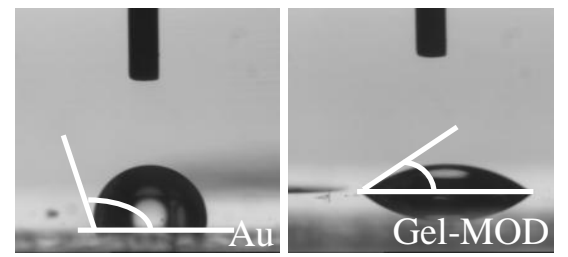

